\title{
Enfermedad cardiovascular y obesidad
}

Artículo / Investigación

Article / Research

Conteúdo / Investigação

\author{
Cardiovascular disease and obesity
}

Doença cardiovascular e obesidade

Darío Saturno

Milagros Castillo

Karen Brea,

karenbrea15@hotmail.com

Francisco Figueroa,

francisco_2380@hotmail.com

Maryori Martínez,

francisco_2380@hotmail.com

Hayde Medina,

haydemedina_17@gmail.com

Nuri Moreno,

nurismorenosp92@gmail.com

Recibido septiembre 2017 / Enviado para modificación diciembre 2017 / Aceptado abril 2018

\section{RESUMEN}

DN: Médico/Especialista. Universidad de Carabobo Facultad de Ciencias de la Salud, Escuela de Medicina. Departamento de Salud Pública. Venezuela.

MC: Msc. Universidad de Carabobo Facultad de Ciencias de la Salud, Escuela de Medicina. Departamento de Salud Pública. Venezuela. KB: Estudiante. Universidad de Carabobo Facultad de Ciencias de la Salud, Escuela de Medicina. Departamento de Salud Pública. Venezuela.

FF: Estudiante. Universidad de Carabobo Facultad de Ciencias de la Salud, Escuela de Medicina. Departamento de Salud Pública. Venezuela.

MM: Estudiante. Universidad de Carabobo Facultad de Ciencias de la Salud, Escuela de Medicina. Departamento de Salud Pública. Venezuela.

HM: Estudiante. Universidad de Carabobo Facultad de Ciencias de la Salud, Escuela de Medicina. Departamento de Salud Pública. Venezuela.

NM: Estudiante. Universidad de Carabobo Facultad de Ciencias de la Salud, Escuela de Medicina. Departamento de Salud Pública. Venezuela.

Introducción: Las enfermedades cardiovasculares constituyen la principal causa de muerte a nivel mundial y en conjunto con la obesidad, tienen gran impacto en la salud pública. Objetivo. Relacionar las enfermedades cardiovasculares y la obesidad, en pacientes que acuden a la consulta de cardiología Valencia/Venezuela, en el. Materiales y Métodos. Se realizó un estudio de campo, descriptivo, con corte transversal. La muestra estuvo conformada por 40 pacientes: Mujeres y hombres entre los 35-60 años de edad, con enfermedades cardiovasculares preexistentes y residentes del estado Carabobo, Venezuela. Los datos se recolectaron a través de un cuestionario tipo ficha clínica, diseñada por los investigadores, acorde a los objetivos del estudio; plasmados en tablas estadísticas, representados en frecuencia relativa y absoluta; con análisis estadístico PAST 3. Resultados: La Hipertensión Arterial, fue la patología con mayor prevalencia, afectando a todos los grupos etarios, de ambos sexos; aunado al estrés y los antecedentes de enfermedades cardiovasculares, como factores de riesgo modificable. En relación al índice de masa corporal predominó el sobrepeso grado II (Pre-obesidad). Conclusión: Existe una alta prevalencia de Hipertensión Arterial, que no respeta edad ni sexo y está estrechamente asociada a la obesidad.

Palabras clave: Enfermedad cardiovascular; Hipertensión Arterial; obesidad, sobrepeso

\section{ABSTRACT}

Introduction. Cardiovascular diseases are the main cause of death worldwide and, in conjunction with obesity, have a great impact on public health. Objective. Relate cardiovascular diseases and obesity in patients attending the Valencia / Venezuela cardiology clinic. Materials and Methods. A descriptive, crosssectional field study was conducted. The sample consisted of 40 patients: women and men between 35-60 years of age, with pre-existing cardiovascular diseases and residents of the state of Carabobo, Venezuela. The data were collected through a questionnaire type clinic, designed by the researchers, according to the objectives of the study; captured in statistical tables, represented in relative and absolute frequency; with statistical analysis PAST 3. Results. Arterial Hypertension was the pathology with the highest prevalence, affecting all age groups, of both sexes; together with stress and a history of cardiovascular diseases, as modifiable risk factors. In relation to the body mass index, overweight grade II (Pre-obesity) predominated. Conclusion. There is a high prevalence of Arterial Hypertension, which does not respect age or sex and is closely associated with obesity.

Key word: Cardiovascular disease; arterial hypertension; obesity; overweight 
RESUMO

Introdução. As doenças cardiovasculares são a principal causa de morte no mundo e, em conjunto com a obesidade, têm grande impacto na saúde pública. Objetivo. Relacionar doenças cardiovasculares e obesidade em pacientes atendidos na clínica cardiológica de Valência / Venezuela. Materiais e métodos. Foi realizado um estudo de campo descritivo, transversal. A amostra foi composta por 40 pacientes: mulheres e homens entre 35-60 anos de idade, com doenças cardiovasculares pré-existentes e residentes no estado de Carabobo, Venezuela. Os dados foram coletados por meio de uma clínica do tipo questionário, elaborada pelos pesquisadores, de acordo com os objetivos do estudo; capturados em tabelas estatísticas, representados em frequência relativa e absoluta; com análise estatística PAST 3. Resultados. A Hipertensão Arterial foi a patologia com maior prevalência, afetando todos os grupos etários, de ambos os sexos; juntamente com o estresse e uma história de doenças cardiovasculares, como fatores de risco modificáveis. Em relação ao índice de massa corporal, predominou o grau de sobrepeso II (Pré-obesidade). Conclusãon. Existe uma alta prevalência de Hipertensão Arterial, que não respeita idade ou sexo e está intimamente associada à obesidade.

Palavras chave: Doença cardiovascular; hipertensão arterial; obesidade; excesso de peso

\section{INTRODUCCIÓN}

$\mathrm{L}$ a Organización Mundial de la Salud (OMS) define la obesidad como la acumulación anormal o excesiva de grasa corporal debido a un desequilibrio entre la ingesta y el gasto de energía, siendo la primera patología crónica no transmisible, donde el consumo de alimentos con alto contenido de grasas y el sedentarismo, ha aumentado la misma, tanto en países desarrollados como en vía de desarrollo. La obesidad es la enfermedad metabólica más frecuente de la actualidad, constituyendo uno de los principales problemas de salud, donde más de mil millones de personas la padecen o tienen sobrepeso; evidenciándose para el 2014 alrededor del 13\% una población adulta mundial con obesidad, afectando en un $11 \%$ a los hombres y en un $15 \%$ a las mujeres (1).

De hecho, estudios epidemiológicos han demostrado que la obesidad es la mayor causa de enfermedad cardiovascular, incluyendo a la enfermedad coronaria, fibrilación auricular, arritmias
DN: Médico/Especialista.

Universidad de Carabobo Facultad de Ciencias de la Salud, Escuela de

Medicina. Departamento de Salud Pública. Venezuela.

MC: Msc. Universidad de Carabobo Facultad de Ciencias de la Salud,

Escuela de Medicina. Departamento de Salud Pública. Venezuela.

KB: Estudiante. Universidad de Carabobo Facultad de Ciencias de la Salud, Escuela de Medicina. Departamento de Salud Pública. Venezuela.

FF: Estudiante. Universidad de Carabobo Facultad de Ciencias de la Salud, Escuela de Medicina. Departamento de Salud Pública. Venezuela.

MM: Estudiante. Universidad de Carabobo Facultad de Ciencias de la Salud, Escuela de Medicina. Departamento de Salud Pública. Venezuela.

HM: Estudiante. Universidad de Carabobo Facultad de Ciencias de la Salud, Escuela de Medicina. Departamento de Salud Pública. Venezuela.

NM: Estudiante. Universidad de Carabobo Facultad de Ciencias de la Salud, Escuela de Medicina. Departamento de Salud Pública. Venezuela. ventriculares y la muerte súbita, pero también se le considera factor causal de Hipertensión Arterial, Diabetes Mellitus II, Enfermedad Articular Degenerativa, Apnea Obstructiva del Sueño, Dislipidemia, Reflujo Gastroesofágico e Hígado Graso no asociado a alcoholismo. Aunado a marcadores de riesgo cardiovascular, que pueden cambiarse, tratarse o modificarse tales como el tabaquismo, la alimentación, obesidad, estrés, alcoholismo, ingesta elevada de sodio, sedentarismo, dislipidemia, mientras que otros no se pueden modificar como la edad, sexo, raza y herencia. Sin embargo, el control de los factores de riesgo, mediante cambios en el estilo de vida o medicamentos, puede reducir el riesgo de padecer enfermedades cardiovasculares (2).

De allí, que en los Estados Unidos 80 millones de personas sufren de Hipertensión Arterial (65 millones) Cardiopatía Coronaria (13 millones); Accidente Cerebrovascular (5,5 millones) y en menor proporción defectos cardiovasculares congénitos e Insuficiencia 
Cardiaca Congestiva (1). También, la tasa de mortalidad en América por enfermedades cardiovasculares fue más alta en Nicaragua, República Dominicana y Trinidad y Tobago, sobrepasando las 200 muertes por cada 100.000 habitantes (3). En México, 7 de cada 10 personas tiene obesidad, siendo la mortalidad 12 veces mayor en jóvenes de 25 a 32 años y el 25\% de las incapacidades laborales son por enfermedades cardiovasculares en un 23\% y Diabetes Mellitus 44\% (1). Asimismo, en Venezuela, más del $20 \%$ de la mortalidad es por enfermedades cardiovasculares, siendo la primera causa de muerte; donde la mayor incidencia $(13,13 \%)$, corresponde a infarto al miocardio, Hipertensión Arterial $(2,24 \%)$ y la enfermedad isquémica $(2,08 \%)(4)$. De allí, que existe una relación indirecta mediada por la Diabetes, Hipertensión Arterial y Dislipidemia, sin embargo, otros refieren que la obesidad es un factor de riesgo independiente de enfermedad cardiovascular (5).

Asimismo, en un estudio sobre la salud cardiovascular de Lara/Venezuela, en 2008 demostró que el factor de riesgo con mayor prevalencia fue el sedentarismo $(47,6 \%)$, más en las mujeres, seguido de la hipertensión arterial ( 1 de cada 3 personas padecían de hipertensión arterial), más en los hombres; mientras que la Diabetes fue el factor de riesgo de menor prevalencia (5\%). Estudio que permitió estudiar la distribución de los factores de riesgo de ECV, en otros lugares de América Latina y El Caribe (6).

De la misma manera, en un estudio observacional y transversal sobre los factores de riesgos cardiovasculares, en pacientes de 4 poblaciones del noroeste del Estado Anzoátegui, Venezuela, en el 20102011, se pudo determinar la prevalencia de los principales factores de riesgo modificables, así como determinar variables como edad, sexo, nivel socioeconómico, observando una alta prevalencia de HTA, seguido de otros factores de riesgo como obesidad, diabetes, hipercolesterolemia, que tiende a aumentar con la edad, siendo más evidente en el sexo femenino (7).

Conviene destacar que, la hipertensión arterial se considera un factor de riesgo cardiovascular; existiendo una clara relación entre las cifras tensiónales elevadas y la aparición de enfermedades cardiovasculares y cerebrovasculares. En ese orden de ideas (8) se realizó un estudio sobre la prevalencia de los factores de riesgo de enfermedad cardiovascular y riesgo cardiovascular en hipertensos, encontrando que el $92,4 \%$ de estos presentaron uno o más factores cardiovasculares modificables asociados, como cuales obesidad y sobrepeso (75,7\%), dislipidemia (57,83\%) y tabaquismo (7,79\%). Se estimó que la prevalencia de síndrome metabólico fue de $61,1 \%$, en la totalidad de los pacientes con riesgo de enfermedad cardiovascular. Sin embargo, el 75,38\% presentaron cifras tensiónales controladas. Se concluyó que 92,4 \% de los hipertensos, tienen uno o más factores de riesgos cardiovascular modificables asociados. La evaluación del riesgo cardiovascular global, se debe en todo hipertenso y a su vez se recomienda la medición del perímetro abdominal, para identificar otros factores de riesgo cardiovascular.

De igual forma (9), en la República de Argentina en un estudio sobre prevalencia $\mathrm{y}$ factores de riesgo bio-socioculturales de obesidad, en adultos de la ciudad de Córdoba. Se concluyó que la alimentación es una necesidad biológica y un hecho social, donde confluyen asuntos de carácter biológico, ecológico, tecnológico, económico, social e ideológico, entre otros; y las asociaciones establecidas entre estos y la obesidad, permitieron establecer factores que protegen $y$ 
promueven el desarrollo de ella y la fuerza con la que se vincula dicha asociación. A lo que se le complementa, (10) un estudio sobre obesidad en personas de 15 y más años en Castilla y León, para describir los indicadores de obesidad en esa población y estudiar su asociación con otros factores de riesgo cardiovascular. Entre los resultados se tuvo que, la prevalencia de obesidad abdominal fue de $36,7 \%$, más en mujeres $(50,1 \%)$, mientras que la proporción de obesidad con IMC $\geq 30$ fue $21,7 \%$, la cual fue mayor en mujeres $(23,2 \%)$, encontrando relación entre la obesidad y otros factores de riesgos cardiovasculares, donde la hipertensión arterial fue superior en obesos $(65,7 \%)$ y en personas con sobrepeso $(45,1 \%)$, que en quienes tenían un IMC normal (21\%). Se concluyó que, la alta prevalencia de obesidad, podría relacionarse con el hecho de que ni la población ni los profesionales de la salud, consideran a la obesidad una enfermedad, sino un factor de riesgo de límites imprecisos, más relacionados con la estética, que con las medidas antropométricas.

Por otra parte, se considera al síndrome metabólico como una asociación de alteraciones del mecanismo de la glucosa, obesidad abdominal, dislipidemia e hipertensión y se ha demostrado que contribuye en la enfermedad cardiovascular (11). De hecho, se realizó un estudio sobre la frecuencia de síndrome metabólico y sus factores asociados, en adultos adscritos a la UMF 1 de IMSS Orizaba Veracruz; obteniendo como resultado que la frecuencia de síndrome metabólico fue $28,08 \%$ sin predominio del sexo, pero con mayor proporción en edades comprendidas entre 50 y 65 años (58,33\%). El 100\% de las personas con obesidad grado I presentaron síndrome metabólico, seguido de pacientes con obesidad grado II y III, 72,91\% y $50 \%$ respectivamente. Se evidenció que $60 \%$ de las personas eran sedentarias y se concluyó que los principales factores asociados al síndrome metabólico fueron la obesidad y el sedentarismo. Las conclusiones fueron que, la frecuencia de SM concuerda con la literatura. Afecta a personas en edades productivas, los factores asociados son modificables, como obesidad y sedentarismo.

Es así, que actualmente, es indiscutible el papel del síndrome metabólico y el de sus componentes, como factores de riesgos para el origen y la progresión de la enfermedad ateroesclerótica y su correlación con la morbimortalidad cardiovascular, así como lo demuestra (12), un estudio sobre la de Prevalencia del Síndrome Metabólico en pacientes con Síndrome Coronario Agudo, en la Unidad de Cuidados Coronarios del Hospital José Carrasco Arteaga, en Cuenca, Perú, en 2009-2010. Entre los resultados se encontró que, la prevalencia del síndrome metabólico fue de $56.50 \%$ (IC 95\%: 52.58\%-60.56\%), más frecuente en el sexo masculino 65\% (IC 95\%: 59.39\%-70.78\%), el 48.50\% (IC 95\%: 43.67\%-53.52\%) entre los 46-64 años. La Angina inestable, fue el síndrome coronario más frecuente con 49.60\% (IC 95\%:45.02\%-54.03\%), seguido del infarto agudo de miocardio, con elevación del segmento ST $33.60 \%$ (IC 95\%:30.53\%-36.85\%) y sin elevación del segmento ST con el $16.80 \%$ (IC 95\%:15.31\%-18.47\%). La obesidad abdominal, se presentó en el $77 \%$ de los casos. Se concluyó que, la prevalencia del síndrome metabólico fue del 56.5\%, predominando en el sexo masculino, entre los 45-64 años de edad. El principal componente del síndrome metabólico, fue el incremento del perímetro abdominal.

El estudio estuvo enmarcado a relacionar las Enfermedades Cardiovasculares y la Obesidad en pacientes que acuden a la consulta de Cardiología de la Ciudad Hospitalaria Dr. 
Enrique Tejera, en el periodo abrilagosto de 2015, Valencia, estado Carabobo en Venezuela; para distribuirlos según edad, sexo, tipo de enfermedad cardiovascular, factores de riesgo cardiovasculares modificables, enfermedades cardiovasculares presentes y clasificar el tipo de obesidad según el índice de masa corporal y su relación con enfermedades cardiovasculares.

El estudio de las Enfermedades Cardiovasculares (ECV) constituye uno de los principales problemas de salud pública en el mundo, ya que representan la primera causa de morbimortalidad. Se acepta que los factores asociados al desarrollo de la ECV, se encuentran relacionados al estilo de vida y determinan el proceso salud-enfermedad ${ }^{6}$. Como se puedo evidenciar en los anteriores argumentos, la obesidad es la mayor causa de muerte de enfermedad cardiovascular, por lo que se considera un problema prioritario, debido al gran número de personas afectadas, su continuo aumento y graves consecuencias sobre la salud. Es una enfermedad crónica, heterogénea, compleja y multifactorial, que ha experimentado un definido incremento de su prevalencia tanto en países desarrollados como los en vías de desarrollo.

\section{MATERIALES Y MÉTODOS}

$\mathrm{S}$ e realizó una investigación de campo con nivel descriptivo (13). Por ser la población un número reducido de pacientes, se tomó su totalidad para la muestra, la cual fue de 40 pacientes, con diagnóstico de enfermedades cardiovasculares, que acudieron a la consulta externa de Cardiología de la Ciudad Hospitalaria Dr. Enrique Tejera, durante el periodo abril-agosto del 2015, de los cuales la muestra estuvo constituida por 40 pacientes voluntarios, con firma de consentimiento informado. Entre mujeres y hombres con diagnóstico de enfermedad cardiovascular, residenciados en el estado Carabobo, con edad mínima de 35 años y máxima de 60 años; siendo el promedio de 48,8 años, con una desviación estándar de $\pm 7,3$; lo que indica que la muestra no está tan dispersa, con respecto al promedio. Según el sexo, la distribución fue proporcional, 20 por cada género; prevaleciendo las edades de 40-44 años, para el sexo masculino con $35 \%(n=7)$ y las edades de 55-60 años, para el sexo femenino, representando un $35 \%(n=7)$. La técnica de recolección de datos, se realizó mediante un instrumento tipo encuesta, tipo ficha clínica diseñada por los investigadores, en base de los objetivos de la investigación, la cual estaba estructurada, en 4 dimensiones:

- Perfil biológico de los pacientes, el cual se divide en dos sub-dimensiones edad y sexo.

- Factores de riesgos cardiovasculares, constituido por la sub-dimensión factores de riesgos modificables.

- Clasificación de las enfermedades cardiovasculares, dividida en 14 subdimensiones: Hipertensión arterial, cardiopatía isquémica, angina de pecho, infarto agudo de miocardio, miocardiopatía, enfermedades valvulares o valvulopatias, cardiopatía reumática, enfermedad pericárdica, arritmias cardiacas, ateroesclerosis, enfermedad cerebrovascular, enfermedad arterial periférica, cardiopatía congénita, paro cardiaco o muerte súbita.

- Medidas antropométricas, que incluye las sub-dimensiones peso, talla, circunferencia abdominal, grosor del pliegue cutáneo e índice de masa corporal.

Luego de elaborado el instrumento, se validó a través de juicio de expertos para seguidamente ser aplicado a la muestra. Una vez aplicado el instrumento $\mathrm{y}$ 
elaborado la recolección de datos, se realizaron los análisis estadísticos, los cuales fueron interpretados a través de frecuencias absolutas y relativas. Todos los datos reunidos del estudio fueron introducidos en la base de datos del programa de análisis estadístico PAST 3, y por último se presentaron cuadros de frecuencia y asociación.

\section{RESULTADOS}

$\mathrm{E}$ n cuanto a las enfermedades cardiovasculares, se observó que la HTA fue la patología que tuvo más registros con un $100 \%(\mathrm{n}=40)$; siendo los más afectados los grupos de 40-44 años, del sexo masculino con registro de $35 \% \mathrm{y}$ de 55-60 años del sexo femenino con un registro de $35 \% \quad(n=7)$, luego la cardiopatía; al registrar para el sexo masculino $35 \%(\mathrm{n}=7)$, con afectación de las clases de 40-44 años, 50-54 años y 5560 años. En cuanto a la angina de pecho, afecto al sexo femenino con registro de 10 $\%(n=2)$ de 55-60 años; para el sexo masculino hubo registro de $10 \%(\mathrm{n}=2)$, afectando solamente los de 40-44 años; la miocardiopatía; afectó a los grupos de 3539 años y el de 55-60 años del sexo femenino con $5 \%(n=1), y$ en el sexo masculino afecto de 40-44 años, con $5 \%$ $(n=1)$. La arritmia cardiaca; con $45 \%(n=9)$ para el sexo femenino, siendo de 45 años a 49 años, el más afectado. Por último, la enfermedad arterial periférica; la cual afectó por igual a ambos sexos, registrando $15 \%(n=3)$ para cada uno, en las edades 40-44 años del sexo masculino y de 45-49 años, en el sexo femenino (Tabla 1).

Tabla 1. Distribución de los pacientes que acuden a la consulta de Cardiología, en la Ciudad Hospitalaria Dr. Enrique Tejera, abril-agosto. Valencia/Venezuela, 2015; según la edad, sexo y tipo de enfermedad cardiovascular.

\begin{tabular}{|c|c|c|c|c|c|c|c|c|c|c|c|c|c|c|c|c|c|c|c|c|c|c|c|c|}
\hline \multicolumn{25}{|c|}{ Enfermedades Cardiovasculares } \\
\hline & \multicolumn{4}{|c|}{ HTA } & \multicolumn{4}{|c|}{ Cardiopatía } & \multicolumn{4}{|c|}{ Angina de Pecho } & \multicolumn{4}{|c|}{ Miocardiopatía } & \multicolumn{4}{|c|}{ Arritmia Cardiaca } & \multicolumn{4}{|c|}{$\begin{array}{l}\text { Enfermedad } \\
\text { Arterial Periférica }\end{array}$} \\
\hline & & $\mathbf{M}$ & & $\mathbf{F}$ & & M & & $\mathbf{F}$ & & M & & $\mathbf{F}$ & & M & & $\mathbf{F}$ & & M & & $\mathbf{F}$ & & M & & $\mathbf{F}$ \\
\hline $\begin{array}{c}35-39 \\
\text { años }\end{array}$ & 4 & $20 \%$ & 1 & $5 \%$ & 1 & $5 \%$ & 1 & $5 \%$ & 0 & $0 \%$ & 0 & $0 \%$ & 0 & $0 \%$ & 1 & $5 \%$ & 0 & $0 \%$ & 1 & $5 \%$ & 0 & $0 \%$ & 0 & $0 \%$ \\
\hline $\begin{array}{l}\text { 40-44 } \\
\text { años }\end{array}$ & 7 & $35 \%$ & 1 & $5 \%$ & 2 & $10 \%$ & 0 & $0 \%$ & 2 & $10 \%$ & 0 & $0 \%$ & 1 & $5 \%$ & 0 & $0 \%$ & 3 & $15 \%$ & 1 & $5 \%$ & 2 & $10 \%$ & 0 & $0 \%$ \\
\hline $\begin{array}{l}45-49 \\
\text { años }\end{array}$ & 2 & $10 \%$ & 6 & $30 \%$ & 0 & $0 \%$ & 0 & $0 \%$ & 0 & $0 \%$ & 0 & $0 \%$ & 0 & $0 \%$ & 0 & $0 \%$ & 1 & $5 \%$ & 3 & $15 \%$ & 0 & $0 \%$ & 2 & $10 \%$ \\
\hline $\begin{array}{c}50-54 \\
\text { años }\end{array}$ & 3 & $15 \%$ & 5 & $25 \%$ & 2 & $10 \%$ & 1 & $5 \%$ & 0 & $05 \%$ & 1 & $5 \%$ & 0 & $0 \%$ & 0 & $0 \%$ & 2 & $10 \%$ & 2 & $10 \%$ & 0 & $0 \%$ & 0 & $0 \%$ \\
\hline $\begin{array}{l}55-60 \\
\text { años }\end{array}$ & 4 & $20 \%$ & 7 & $35 \%$ & 2 & $10 \%$ & 3 & $15 \%$ & 0 & $0 \%$ & 2 & $10 \%$ & 0 & $0 \%$ & 1 & $5 \%$ & 0 & $0 \%$ & 2 & $10 \%$ & 1 & $5 \%$ & 1 & $5 \%$ \\
\hline Total & 20 & $100 \%$ & 20 & $100 \%$ & 7 & $35 \%$ & 5 & $25 \%$ & 2 & $10 \%$ & 3 & $15 \%$ & 1 & $5 \%$ & 2 & $10 \%$ & 6 & $30 \%$ & 9 & $45 \%$ & 3 & $15 \%$ & 3 & $15 \%$ \\
\hline
\end{tabular}

Según la distribución de los pacientes por los factores de riesgo modificables, el estrés y los antecedentes de enfermedades cardiovasculares predominaron con $75 \%$
( $n=30)$, seguido del sedentarismo que se presentó en un 72,5 \% ( $\mathrm{n}=29)$. El tabaquismo, se tuvo menor incidencia con $17,5 \quad \% \quad(n=7) . \quad($ Tabla 2$)$. 
De igual forma, la HTA fue la enfermedad cardiovascular, que prevaleció con $100 \%$ $(\mathrm{n}=40)$, luego las arritmias cardiacas con
$37,5 \%(\mathrm{n}=15)$ y después la cardiopatía con $30 \%(n=12)$.

Tabla 2. Distribución de los factores de riesgo modificables, en la Ciudad Hospitalaria Dr. Enrique Tejera, abril-agosto. Valencia/Venezuela, 2015

\begin{tabular}{lcc}
\hline Factor de Riesgo & f & \% \\
\hline Estrés & 30 & $75 \%$ \\
AEC & 30 & $75 \%$ \\
Sedentarismo & 29 & $72,5 \%$ \\
AF-Obesidad & 24 & $60 \%$ \\
Alcohol & 17 & $42,5 \%$ \\
Tabaquismo & 7 & $17,5 \%$ \\
\hline
\end{tabular}

Con respecto al índice de masa corporal y según la clasificación utilizada, prevaleció el sobrepeso grado II (Pre-obesidad) que correspondió al rango de $27-29,9 \mathrm{~kg} / \mathrm{mts}^{2}$, con un valor de $35 \%(\mathrm{n}=14)$, luego la obesidad tipo I, con un índice de masa corporal en el rango de $30-34,9 \mathrm{~kg} / \mathrm{mts}^{2}$, que reportó $20 \%(\mathrm{n}=8)$ del total de la muestra. En este estudio, no hubo pacientes con peso insuficiente según el IMC ni con obesidad de tipo IV (extrema). (Tabla 3).

Tabla 3. Distribución de las Enfermedades Cardiovasculares, en la Ciudad Hospitalaria Dr. Enrique Tejera, abril-agosto. Valencia/Venezuela, 2015

\begin{tabular}{lcc}
\hline Enfermedades Cardiovasculares & & \\
\hline HTA & f & $\%$ \\
\hline Arritmias cardiacas & 40 & $100 \%$ \\
\hline Cardiopatía & 15 & $37,5 \%$ \\
\hline Enfermedad arterial periférica & 12 & $30 \%$ \\
\hline Angina de pecho & 6 & $15 \%$ \\
\hline Miocardiopatía & 5 & $12,5 \%$ \\
\hline
\end{tabular}

Finalmente, se observó que en la totalidad de los pacientes con pre-obesidad $35 \% \quad(\mathrm{n}=14)$, predominó la HTA, en conjunto con la arritmia cardiaca $10 \%$ $(n=4)$, y la cardiopatía $10 \%(n=4) ;$ seguido de los pacientes con obesidad tipo I, en los cuales predominó en un $20 \%(\mathrm{n}=8)$ con HTA en conjunto con arritmia cardiaca $10 \%(\mathrm{n}=4)$. (Tabla 4$)$ 
Tabla 4. Distribuidos según índice de masa corporal en la Ciudad Hospitalaria Dr. Enrique Tejera, abril-agosto. Valencia/Venezuela, 2015

Índice de Masa Corporal

\begin{tabular}{lcc}
\hline$\left(\mathrm{kg} / \mathrm{mts}^{2}\right)$ & f & $\mathbf{\%}$ \\
\hline Peso insuficiente $\mathbf{1} \mathbf{1 8 , 5}$ & & \\
\hline Normopeso $\mathbf{1 8 , 5} \mathbf{- 2 4 , 9}$ & 7 & $17,5 \%$ \\
\hline Sobrepeso grado I $\mathbf{2 5}-\mathbf{2 6 , 9}$ & 5 & $12,5 \%$ \\
\hline Sobrepeso grado II (Preobesidad) $\mathbf{2 7}-\mathbf{2 9 , 9}$ & 14 & $35 \%$ \\
\hline Obesidad de tipo I 30 -34,9 & 8 & $20 \%$ \\
\hline Obesidad de tipo II 35- 39,9 & 4 & $10 \%$ \\
\hline Obesidad de tipo III (Mórbida) 40- 49,9 & 2 & $5 \%$ \\
\hline Obesidad de tipo IV (Extrema) $>\mathbf{5 0}$ & & \\
\hline Total & 40 & $100 \%$ \\
\hline
\end{tabular}

\section{CONCLUSIONES}

$\mathrm{L}$ a Hipertensión Arterial es una patología con gran incidencia, en ambos sexos y edades. Siendo el estrés y los antecedentes y enfermedades cardiovasculares, la segunda escala de factores de riesgo modificables según el sexo. Con un índice de masa corporal, donde resaltó el sobrepeso grado II (Preobesidad). De allí, que la obesidad sea considerada un problema de salud pública, en muchos países y en Venezuela, lo que conlleva a intensificar una estrategia poblacional, para reducir el consumo de tabaco, aumentar la actividad física y reforzar los hábitos alimenticios sanos. Además, es importante un adecuado control de la hipertensión arterial y los factores asociados como dislipidemias y diabetes; por medio de una prevención primaria, que eduque a la población, sobre una alimentación sana y balanceada, con el consumo mínimo de grasas, comida chatarra y carbohidratos, para así poder evitar la obesidad.

También, se debe evitar el consumo de tabaco y alcohol, para disminuir todo tipo de enfermedad cardiovascular.

Por lo tanto, al aparecer la obesidad, se tienen problemas de hipertensión arterial $y$ se diagnostica cualquier tipo de enfermedad cardiovascular, lo que nos obliga a fomentar un buen control por especialistas ya sea cardiólogo y/o internista, para individualizar un tratamiento adecuado y evitar complicaciones, así como insistir en un buen estilo de vida.

\section{REFERENCIAS}

1. Organización mundial de la Salud (OMS) [Página de internet]. Fuente OPS. Enfermedades cardiovasculares en las Américas. Chile. 2007 [Citado 12/3/15]. Disponible en: http://www.paho.org/chi/index.php?option=com_co ntent\&view=article\&id $=172 \&$ Itemid $=1$

2. Organización Mundial de la Salud (OMS) [Página de internet]. Enfermedades cardiovasculares. Estados Unidos. 2015 [Citado 12/3/15]. Disponible en:

http://www.who.int/cardiovascular_diseases/about_ $\mathrm{cvd} / \mathrm{es} /$

3. Organización Mundial de la Salud (OMS) [Página de internet]. Enfermedades Cardiovasculares. Estados Unidos. 2013 [Citado 12/3/15]. Disponible en: 
4. http://www.who.int/mediacentre/factsheets/fs317/es I

5. Anuario de mortalidad 2011 [Página de internet]. Venezuela. 2014 [Citado 12/3/15]. Disponible en: file://C:/Users/Extreme/Downloads/Anuario2011\% 20(1).pdf

6. López F, Cortes M. Obesidad y Corazón. Revista Española de Cardiología [Revista en internet]. España. 2011 [Citado 14/3/15]; 64 (2). Disponible en: http://www.revespcardiol.org/es/obesidadcorazon/articulo/13191034/

7. Infante E, Navarro A, Finizola M, Zevallos J, Moya D, Alvarado S y Finizola B. Estudio de la salud cardiovascular en el Estado Lara (escel 2008): metodología del proyecto y perfil de la muestra estudiada. Avances Cardiol [Revista en Internet] 2010; 30 (4):316-325. Venezuela; Octubre, 2010. [Citado 3/11/14]. Disponible en: http://svcardiologia.org/es/images/documents/Avan ce_Cardiologico/art_vol_30_2010/Vol_30_4_2010/ 04.InfanteE316325.pdf

8. Rojas E. Prevalencia de factores de riesgo cardiovascular en 4 poblaciones rurales del noroeste de Estado Anzoátegui. Revista

9. Latinoamericana de Hipertensión [Revista de internet]. 2012; [Citado 3/

10. 11/14] 7 (2): 35-39 Disponible en: http://www.revistahipertension.com/rlh_7_2_2012/p revalencia_factores.pdf

11. Moreno L. Prevalencia de los Principales Factores de Riesgos de Enfermedad Cardiovascular y Riesgo Cardiovascular en Paciente con Hipertensión Arterial que asisten a una IPS en Bogotá y algunos municipios de Cundinamarca. [Tesis de internet]. Colombia: Pontificia Universidad Javeriana; diciembre 2009. [Citado 3/11/14]. Disponible en:http://www.javeriana.edu.co/biblos/tesis/medicin a/tesis39.pdf

12. Aballay L. La obesidad en córdoba: Estudio de su Prevalencia e Identificación de Factores de Riesgo. [Tesis de internet]. República de Argentina: Universidad Nacional de Córdoba; 2012. [Citado 3/11/14]. Disponible en:

http://rdu.unc.edu.ar/bitstream/handle/11086/714/.A BALLAY.pdf?sequence=1García $S$, Vegas $T$, Lozano J, Álamo R, Lleras S, Castrodeza J "ET $A L$ ". Patrón epidemiológico de la obesidad en Castilla y León y su relación con otros factores de riesgo de enfermedad cardiovascular.

13. Rev Sociedad Española de Cardiología. [Revista de internet]. Enero 2011; 64 (1): 63-66 Disponible: http://www.revespcardiol.org/es/content/articulo/13 190102/

14. Benítez U. Frecuencia de Síndrome Metabólico: factores asociados. [Tesis de Internet]. México: Universidad Veracruzana; 2010. [Citado 3/11/14]. Disponible en: http://cdigital.uv.mx/bitstream/123456789/32321/1/ mateos\%20benitez.pdf

15. Ortega J. Prevalencia del Síndrome Metabólico en Pacientes con Síndrome Coronario Agudo en el Hospital José Carrasco Arteaga Cuenca 20092010. [Tesis de Internet]. Ecuador: Universidad de Cuenca; 2010. [Citado 3/11/14].
Disponibleen:http://dspace.ucuenca.edu.ec/jspui/bit stream/123456789/3956/1/MEDMI09.pdf

16. Fidias G Arias. Proyecto de investigación: Guía para su elaboración. 3era Ed. Caracas: Episteme; 1999.

17. López F Jiménez, Cortez M Bergoderi. La obesidad y el corazón. [Revista en internet] 2011. [Citado 3/11/14] Disponible en: http://www.revespcardiol.org/es/obesidadcorazon/articulo/13191034/ 